\title{
Study of fetomaternal outcomes in antepartum haemorrhage at tertiary care centre
}

\author{
Faaizah Ghanchibhai*, Sharda Goyal, Nalini Sharma, Ankita Pargee
}

Department of Obstetrics and Gynecology, GMCH, Udaipur, Rajasthan, India

Received: 20 January 2021

Accepted: 06 March 2021

\section{*Correspondence:}

Dr. Faaizah Ghanchibhai,

E-mail: faaizah.3722@gmail.com

Copyright: (c) the author(s), publisher and licensee Medip Academy. This is an open-access article distributed under the terms of the Creative Commons Attribution Non-Commercial License, which permits unrestricted non-commercial use, distribution, and reproduction in any medium, provided the original work is properly cited.

\begin{abstract}
Background: Antepartum haemorrhage (APH) is a challenge to obstetrician as it involves a question of life and death to mother and fetus. Antepartum hemorrhage is the 2 nd most common cause of maternal mortality and morbidity as sepsis and obstructed labour has decreased now. The aim of the study is to find the prevalence of APH. And to study foeto-maternal outcomes in patients with antepartum haemorrhage, association of comorbidities and risk factors.

Methods: This study was conducted at Geetanjali Medical College and Hospital, Udaipur after obtaining approval from institutional research ethical board and written informed consent during the period of February 2019 to July 2020. This was prospective observational study, sample size was 60 patients. All the APH patients who were admitted at GMCH Obstetrics and Gynaecology department after 28 weeks of gestation were included in study. Extrauterine or bleeding due to general pathology was excluded. Total number of delivery were 1900 in above duration and number of APH patients were 60 , so our incidence is $3.1 \%$.

Results: Total number of delivery were 1900 in above duration and number of APH patients were 60, so our incidence is 3.1\%. According to maternal complications rate, It was $66.66 \%$ in abruptio placenta, $37.03 \%$ in placenta previa and jointly $53.33 \%$. In placenta previa group $100 \%$ patients discharged with good GC, In abruptio placenta group $96.96 \%$ patients discharged with good GC and 3.04\% patients discharged with poor GC. Perinatal mortality was zero in placenta previa group and in abruptio placenta, it was $27.27 \%$ (24.24\% IUD, 3.03\% neonatal death).

Conclusions: The main cause of APH was abruption which was seen in $33(55 \%)$ of patients and placenta previa was seen in $27(45 \%)$ of patients. Maternal and neonatal complications, both were very high in abruption group as compared to placenta previa group. Overall maternal mortality rate was $53.33 \%$ and perinatal mortality rate was zero in placenta previa group while in abruption placenta group, it was $27.27 \%$. There was no maternal complications in booked placenta previa cases only there were very few complications in emergency cases. In abruptio placenta group also complications were more in emergency cases, irregular booked and uncontrolled pregnancy pathology cases.
\end{abstract}

Keywords: Abruption placenta, Antepartum hemorrhage, Placenta previa

\section{INTRODUCTION}

Pregnancy is a unique, exciting and joyous time in a woman's life, as it highlights woman's creative and nurturing powers while providing a bridge to the future. It is spiritually uplifting and often challenging, because a pregnant woman needs also to be a responsible woman so as to best support the health of her future child.
Antepartum hemorrhage is a challenge to obstetrician as it involves a question of life and death to mother and fetus. Maternal mortality ratio in India is 113 per $1,00,000$ live births and worldwide it is 211 per $1,00,000$ live births. ${ }^{1,2}$ Various maternal mortality causes are hemorrhage (APH, PPH), infection, hypertensive disorders of pregnancy, unsafe abortion, obstructed labour, anemia, viral hepatitis and other medical 
disorders. They may lead to severe maternal morbidity and mortality. APH is second major cause of maternal mortality as sepsis and obstructed labour has decreased now. Perinatal mortality rate in India is reported to be 26 per 1000 births. $^{3}$ APH is responsible for about $10 \%$ of perinatal deaths.

Any bleeding from or into the genital tract during pregnancy, after the period of viability (22-24 weeks of gestation) till the delivery of the fetus (end of the second stage) is defined as antepartum hemorrhage. It occurs in $2-5 \%$ of pregnancies. ${ }^{4}$ About one-third of APH belongs to placenta previa, its incidence ranges from 0.5 to $1 \% .^{5}$ Abruptio placenta incidence is about 1 in 100 deliveries. $^{6}$ Depending on the extent and intensity of placental separation, it is significant cause of maternal mortaliy (2$5 \%)$ and perinatal mortality $(15-20 \%)$. APH is an important cause of foeto maternal mortality and morbidity. Various maternal complications due to APH are, excessive bleeding causing shock, preterm labour, blood coagulation disorders like DIC, acute renal failure, morbidly adherent placenta, PPH, puerperal sepsis, lactation failure, pulmonary embolism. Fetal complications are prematurity, asphyxia, intrauterine foetal death and birth injuries.

APH is mainly due to Placenta previa (low lying placenta in lower segment) or Abruptio placenta (premature placental separation or accidental hemorrhage). Sometimes it is due to local causes like cervico-vaginal lesions- cervical polyp, Ca cervix, varicose vein and local trauma. Rarely it is unexplained.

Predisposing factors of placenta previa are advanced maternal age, multiparity, multiple pregnancy, previous placenta previa, deficient endometrium due to presence of uterine scar like previous caesarean section and previous curettage, endometritis, manual removal of placenta, smoking and ART procedures. Predisposing factors of accidental hemorrhage are advanced maternal age, multiparity, preeclampsia, chronic hypertension, prior abruption, chorioamnionitis, poor socio-economic condition, malnutrition, cigarette smoking, cocaine use, multifetal gestation, trauma, short cord, placental anomaly, torsion of uterus, uterine leiomyoma. ${ }^{7}$

Our hospital is a multispecialty and super speciality tertiary referred centre and attached to a medical college, hence we get lots of complicated cases of APH and these are treated jointly by Obstetrics and Gynaecology department as well as other super speciality departments like, Nephrology, Gastroenterology, Neurology. Hence I have done this study to know the incidence of APH, its complications and to give suggestions for better, timely management, So, foeto maternal mortality and morbidity can be reduced.

Aim of the study was to study foeto-maternal outcomes in patients with antepartum haemorrhage.
Primary objectives was to assess the maternal outcome with APH in terms of development of toxaemia, anemia, preterm labour, shock, HELLP SYNDROME, DIC, PPH, obstetric hysterectomy, admission to ICU and maternal morbidity and mortality. To assess the foetal outcome with APH. To study risk factors of APH. To study the prevalence of antepartum haemorrhage at tertiary care hospital.

Secondary objectives of the study was to study the association of comorbidities and high-risk factors with APH like anaemia, previous history of curettage, previous caesarean section, preeclampsia, malpresentation, adherent placenta.

\section{METHODS}

\section{Study design}

This was a prospective study of all the patients of third trimester pregnancy with APH admitted during the period of February 2019 to July 2020 at Geetanjali Hospital, Udaipur, a tertiary care hospital.

\section{Inclusion criteria}

All cases of antepartum hemorrhage $>28$ weeks.

\section{Exclusion criteria}

Bleeding from a source other than uterus. Patient suffering from any other bleeding disorder.

\section{Methodology}

After obtaining written informed patient consent, this study was conducted at Geetanjali Medical College and Hospital Udaipur from January 2019 to July 2020. On admission, a complete history including history of present complaint, obstetric history, menstrual history, past history, family history was taken. Information regarding her age, address, socioeconomic status and dietary habits, smoking drug use, history regarding her previous antenatal checkups were noted. General physical examination, abdominal examination, per speculum and per vaginum examination (when required) were done to assess both maternal and fetal condition. All patients presenting with APH were initially investigated and managed as outlined below, but subsequent management was determined according to the suspected cause, severity and type of bleeding and the gestational age of the pregnancy. The initial management included the following:

Intravenous fluids were given according to severity of bleeding and patient's general condition. Blood samples were obtained for immediate hemoglobin and hematocrit estimation, complete blood count and for grouping and cross matching. USG was done to establish the cause of APH, when maternal and fetal conditions stablize. After 
this initial management, further management based on following criteria:

According to GC of the patient, Bleeding present or not, The bleeding was continuing but remained mild and nonlife threatening, The amount of bleeding and whether it was severe and life threatening or not, FHS present or not, If there was fetal demise or not. The subsequent management, based on the above factors was divided into,

\section{Active management}

Indications, Maternal GC- poor, continue bleeding, fetal death, term pregnancy

\section{Expectant management}

Indications, Maternal GC-good, bleeding stopped, GA< 34 weeks, FHS good. These were carried out according to the suspected cause of APH, based on the history, examination and investigations. If Placenta previa and Abruptio placenta were excluded, rest of the patients with APH were placed under Unclassified hemorrhage (UH) and further management 40 depended on gestational age, nature of bleeding (persistent or recurrent and severity), the state of the fetus (absence or presence of fetal distress and the cause) and cause of bleeding.

\section{RESULTS}

Table 1: Distribution of patients according to diagnosis.

\begin{tabular}{|lll|}
\hline Diagnosis & No. & $\%$ \\
\hline Abruptio placenta & 33 & 55.00 \\
\hline Placenta previa & 27 & 45.00 \\
\hline Total & 60 & 100 \\
\hline
\end{tabular}

This study was conducted at Geetanjali Medical College and Hospital, Udaipur after obtaining approval from institutional research ethical board and written informed consent during the period of February 2019 to July 2020.

This was prospective observational study, sample size was 57 patients, we have taken 60 patients. All the APH patients who were admitted at GMCH Obstetrics and Gynaecology department after 28 weeks of gestation were included in study. Extrauterine or bleeding due to general pathology was excluded. Total numberof delivery were 1900 in above duration and number of APH patients were 60 , So our incidence is $3.1 \%$.

Table 1 shows, 33 (55\%) cases of abruptio placenta group of patients, 27 (45\%) of placenta previa group of patients. Table 2 shows, in placenta previa group booked $(70.37 \%)$ pateints were more as compared to unbooked $(29.63 \%)$ patients, but in the abruptio placenta group unbooked $(57.58 \%)$ patients were more as compared to booked $(42.42 \%)$ patients. Table 3 shows, commonest factors in both groups were advanced maternal age and ARTabruptio placenta (21.21\%) and placenta previa (29.62\%), other predisposing factors in placenta previa group were previous curettage (33.33\%), previous CS (37\%), anaemia (14.81\%), pre-eclampsia $(7.41 \%)$, GHTN $(3.7 \%)$, while in abruptio placenta group- pre eclampsia (54.44\%), Anemia (27.27\%), GHTN (6.06\%) previous CS $(9.09 \%)$ were present. According to maternal complications rate (Table 4), It was $66.66 \%$ in abruptio placenta, $37.03 \%$ in placenta previa and jointly $53.33 \%$. In placenta previa group, main maternal complication was PPH $29.63 \%, 22.22 \%$ of them had shock, $3.7 \%$ had obstetric hysterectomy and $37.04 \%$ had blood transfusion. In abruptio placenta also main complication was PPH $57.58 \%$, shock $33.33 \%$, couvelaire uterus $21.21 \%$, obstetric hysterectomy $3.7 \%$, rest complications were DIC, ARF, HELLP syndrome in same patient $3.03 \%$ and $66.67 \%$ had blood transfusion also. Table 5 shows In placenta previa group $100 \%$ patients discharged with good GC, in abruptio placenta group $96.96 \%$ discharged with good GC and 3.04\% discharged with poor GC. And there was no death in both the group.

Table 2: Distribution of patients according booking status of the patients.

\begin{tabular}{|lllllll|}
\hline & \multicolumn{2}{c}{ Abruptio placenta $(\mathbf{n = 3 3 )}$} & \multicolumn{2}{c|}{ Placenta previa $(\mathbf{n = 2 7})$} & \multicolumn{2}{c|}{ Total } \\
& No. & $\mathbf{\%}$ & No. & \% & No. & \% \\
\hline Booked & 14 & 42.42 & 19 & 70.37 & 33 & 55.00 \\
\hline Unbooked & 19 & 57.58 & 8 & 29.63 & 27 & 45.00 \\
\hline
\end{tabular}

Table 3: Distribution of patients according to predisposing factors.

\begin{tabular}{|llll|lll|}
\hline \multirow{2}{*}{ Predisposing factors } & Abruptio placenta $(\mathbf{n = 3 3})$ & \multicolumn{2}{c|}{ Placenta previa $(\mathbf{n = 2 7})$} & \multicolumn{2}{c|}{ Total $(\mathbf{n = 6 0})$} \\
\cline { 2 - 8 } & No. & $\%$ & No. & \% & No. & \% \\
\hline ART & 3 & 9.09 & 4 & 14.81 & 7 & 11.67 \\
\hline Advanced maternal age & 4 & 12.12 & 4 & 14.81 & 8 & 13.33 \\
\hline Anaemia & 9 & 27.27 & 4 & 14.81 & 13 & 21.67 \\
\hline G.HTN & 2 & 6.06 & 1 & 3.70 & 3 & 5.00 \\
\hline Pre-eclampsia & 18 & 54.55 & 2 & 7.41 & 20 & 33.33 \\
\hline H/O curettage & - & 0.00 & 9 & 33.33 & 9 & 15.00 \\
\hline
\end{tabular}




\begin{tabular}{|llll|lll|}
\hline \multirow{2}{*}{ Predisposing factors } & \multicolumn{2}{c}{ Abruptio placenta $(\mathrm{n}=\mathbf{3 3})$} & \multicolumn{2}{c|}{ Placenta } & previa $(\mathrm{n}=\mathbf{2 7})$ & \multicolumn{2}{c|}{ Total $(\mathrm{n}=\mathbf{6 0})$} \\
\cline { 2 - 7 } & No. & $\%$ & No. & $\%$ & No. & $\%$ \\
\hline H/O placenta previa & 0 & 0.00 & 1 & 3.70 & 1 & 1.67 \\
\hline Previous CS & 3 & 9.09 & 10 & 37 & 13 & 21.67 \\
\hline
\end{tabular}

Table 4: Distribution of patients according to maternal complications.

\begin{tabular}{|lllll|}
\hline \multirow{2}{*}{ Complications } & \multicolumn{2}{c}{ Abruptio placenta $(\mathbf{n = 3 3})$} & \multicolumn{2}{c|}{ Placenta Previa $(\mathbf{n = 2 7})$} \\
\hline PPH & No. & $\mathbf{\%}$ & No. & $\mathbf{\%}$ \\
\hline Shock & 19 & 57.58 & 8 & 29.63 \\
\hline DIC & 11 & 33.33 & 6 & 22.22 \\
\hline ARF & 1 & 3.03 & 0 & 0.00 \\
\hline HELLP syndrome & 1 & 3.03 & 0 & 0.00 \\
\hline Couvelaire uterus & 1 & 3.03 & 0 & 0.00 \\
\hline Obstetric hysterectomy & 7 & 21.21 & 0 & 0.00 \\
\hline ICU admission & 1 & 3.03 & 1 & 3.70 \\
\hline Blood Transfusion & 13 & 39.39 & 6 & 22.22 \\
\hline
\end{tabular}

Table 5: Distribution of patients according to final maternal outcome.

\begin{tabular}{|c|c|c|c|c|}
\hline \multirow{2}{*}{ Maternal outcome } & \multicolumn{2}{|c|}{ Abruptio placenta (n=33) } & \multicolumn{2}{|c|}{ Placenta previa (n=27) } \\
\hline & No. & $\%$ & No. & $\%$ \\
\hline Discharged with Fair GC & 32 & 96.96 & 27 & 100 \\
\hline Discharged with Poor GC & 1 & 3.04 & 0 & 0 \\
\hline Death & 0 & 0 & 0 & 0 \\
\hline
\end{tabular}

Table 6: Distribution of patients according to neonatal outcome.

\begin{tabular}{|llllll|}
\hline \multirow{2}{*}{ Neonatal outcome } & & \multicolumn{2}{c}{ Abruptio placenta $(\mathbf{n = 3 3})$} & \multicolumn{2}{l|}{ Placenta previa $(\mathbf{n = 2 7})$} \\
\cline { 2 - 6 } & & No. & $\mathbf{\%}$ & No. & \% \\
\hline \multirow{3}{*}{ Apgar Score } & $<3$ & 8 & 24.24 & 0 & 0.00 \\
& $3-7$ & 3 & 9.09 & 0 & 0.00 \\
\cline { 2 - 6 } & $>7$ & 22 & 66.67 & 27 & 100.00 \\
\hline \multirow{2}{*}{ Birth weight } & $<2.5$ & 29 & 87.88 & 14 & 51.85 \\
\cline { 2 - 6 } & $\geq 2.5$ & 4 & 12.12 & 13 & 48.15 \\
\hline \multirow{2}{*}{ Outcome } & Alive & 30 & 90.91 & 27 & 100 \\
\cline { 2 - 6 } & IUD & 8 & 24.24 & 0 & 0.00 \\
\hline \multirow{2}{*}{ NICU Admission } & Yes & 15 & 45.45 & 8 & 29.63 \\
\cline { 2 - 6 } & No & 18 & 54.55 & 19 & 70.37 \\
\hline
\end{tabular}

Table 7: Distribution of patients according to final neonatal outcome.

\begin{tabular}{|lllll|}
\hline Final outcome & \multicolumn{2}{l}{ Abruptio placenta $(\mathbf{n}=\mathbf{3 3})$} & \multicolumn{2}{l|}{ Placenta previa $(\mathbf{n}=\mathbf{2 7})$} \\
\hline Discharged & No. & $\mathbf{\%}$ & No. & $\mathbf{\%}$ \\
\hline Death & 24 & 72.73 & 27 & 100.00 \\
\hline
\end{tabular}

Table 6 shows neonatal outcome. In placenta previa group apgar score was $>7(100 \%)$ in all the neonates, birth weight $\geq 2.5 \mathrm{~kg}(51.85 \%)$ and $<2.5 \mathrm{~kg}(48.15 \%)$, all were alive and NICU admission was $29.63 \%$. In abruptio placenta group, apgar score $(<3-24.24 \%, 3-7-9.09 \%,>7-$ $66.67 \%)$, birth weight $\geq 2.5 \mathrm{~kg}(12.12 \%)$ and $<2.5 \mathrm{~kg}$
(87.88\%). $24.24 \%$ were not alive, NICU admission was $45.45 \%$ of neonates.

Table 7 shows, in placenta previa group $100 \%$ neonates were discharged while in abruptio placenta group only $72.73 \%$ neonates were discharged and rest had perinatal mortality (24.24\% IUD, 3.03\% neonatal death). 


\section{DISCUSSION}

Total number of APH patients were 60, Out of this, abruptio placenta group of patients were $55 \%$ and placenta previa group of patients were $45 \% . \mathrm{K}$ Lakshmipriya et al (2019) found that incidence of abruptio placentae was $56 \%$ and placenta previa was $44 \%$, which is similar to our study. ${ }^{12}$ According to booking status, In placenta previa group maximum number of patients $19(70.37 \%)$ were booked, While in Abruptio placenta group maximum number of patients 19 $(57.58 \%)$ were unbooked. K Lakshmipriya et al (2019) found in their study that $93.3 \%$ patients were booked as compared to $6.7 \%$ patients who were unbooked. ${ }^{12}$ According to predisposing factors, commonest factors in both groups were advanced maternal age $(13.33 \%)$ and ART(11.67\%), other predisposing factors in placenta previa group were previous curettage $(33.33 \%)$,previous LSCS $(36 \%)$, anemia $(14.81 \%)$, pre eclampsia $(7.41 \%)$, GHTN $(3.7 \%)$, While in abruptio placenta group- pre eclampsia $(54.55 \%)$, anemia $(27.27 \%)$, G.HTN $(6.06 \%)$, previous LSCS $(9.09 \%)$ were present. Chandnani K et al (2019), Wasnik et al (2015), Majumder S et al (2015) found that cause of placenta previa was previous history of curettage in $33.2 \%, 52 \%, 12.12 \%$ respectively. ${ }^{13,8,9}$ Majumder S et al (2015) found that anaemia was found in $75 \%$ of patients and pre-eclampsia in $22 \%, 73 \%$ cases were associated with pregnancy induced hypertension. ${ }^{9} \mathrm{~K}$ Lakshmipriya et al (2019) found that $73 \%$ cases had history of previous LSCS. ${ }^{12}$ According to maternal complications rate, It was $66.66 \%$ in abruptio placenta, $37.03 \%$ in placenta previa and jointly $53.33 \%$. In placenta previa group, main maternal complication was PPH 29.63\%, shock 22.22\%, obstetric hysterectomy $3.7 \%$, need of blood transfusion in $37.04 \%$. In abruptio placenta group also main complication was PPH 58\%, shock $33.33 \%$, couvelaire uterus $21.21 \%$, obstetric hysterectomy $3.03 \%$, need of blood transfusion in $66.67 \%$ other complications were DIC, ARF, HELLP syndrome in one patient $3.03 \%$.

Indications of obstetric hysterectomy : placenta accrete in placenta previa and abruption placenta, it was uncontrolled PPH due to coagulation failure.

Wasnik et al (2015) found that $75 \%$ of patients required blood transfusion. The very high rates of blood transfusion in present study might be due to the reason that more number of patients were having anemia and had PPH. ${ }^{8}$ Tyagi $\mathrm{P}$ et al (2016) found that PPH was a major intrapartum complication involving $45 \%$ cases of APH, $42.5 \%$ cases of placenta previa and $57 \%$ cases of abruptio placenta. ${ }^{10}$ So PPH percentage in abruption placenta is same but in placenta previa it is less in our study. Caesarean hysterectomy was performed in $7 \%$ APH, 5\% placenta previa and $2 \%$ abruptio placenta, in our study also total percentage of hysterectomy was $7 \%$ which is similar to Chandnani K et al (2019) found that in abruptio placenta incidence of DIC was $4.7 \%$ and couvelaire uterus in $5 \%$ cases. ${ }^{13}$ Kapadia L et al (2017) found that PPH (11\%), DIC (16\%), ARF (6\%), Shock $(9 \%)$ patients. $^{11}$

Final maternal outcome was also very good, In placenta previa group $100 \%$ discharged with good GC, In abruptio placenta group $96.96 \%$ discharged with good GC and $3.04 \%$ discharged with poor GC, it was same patient who had coagulation failure. There was no death in both the groups. In Wasnik et al (2015) study also there was no mortality. ${ }^{8}$ According to Neonatal outcome, In placenta previa group apgar score was $>7(100 \%)$ in all the neonates, birth weight $\geq 2.5 \mathrm{~kg}(51.85 \%)$ and $<2.5$ $\mathrm{kg}(48.15 \%)$, all were alive and NICU admission was $29.63 \%$. Perinatal mortality was zero. In abruptio placenta group, apgar score $(<3-24.24 \%, 3-7-9.09 \%,>7$ $-66.67 \%)$, birth weight $\geq 2.5 \mathrm{~kg}(12.12 \%)$ and $<2.5 \mathrm{~kg}$ (87.88\%), 24.24\% were IUD, admitted as IUD. NICU admission was $45.45 \%$, neonatal death was $3.03 \%$. Perinatal mortality in abruption placenta group was $27.27 \%$.

Our perinatal mortality was zero in placenta previa group due to timely proper management of the patient and more number of booked patient. In abruptio placenta also we had only $27.27 \%$ perinatal mortality though the emergency patients were more, because of proper, timely management and well equipped NICU. Majumder S et al (2015) Perinatal mortality in $44.1 \%$ cases of abruptio placenta and $12.1 \%$ cases of placenta previa. $^{9}$ Perinatal loss is higher in babies with low birth weight and higher number of cases of abruption during 33-37 weeks explains high perinatal mortality rate in babies with birth weight $<2.5 \mathrm{~kg}$. Wasnik et al (2015) $40 \%$ of babies were low birth weight. ${ }^{8}$ Perinatal mortality was $21 \%$, $(24.44 \%$ in placenta previa and $19.23 \%$ in abruptio placenta group.

According to final neonatal outcome, in placenta previa group $100 \%$ neonates were discharged while in abruptio placenta group only $72.73 \%$ neonates were discharged and rest had perinatal mortality $(24.24 \%$ IUD, $3.03 \%$ neonatal death).

\section{CONCLUSION}

Conclusion of this study is that maternal and neonatal complications, both were very high in abruptio placenta group as compared to placenta previa group. Overall maternal morbidity rate was $53.33 \%$ and perinatal mortality rate was zero in placenta previa group while in abruptio placenta group, it was $27.27 \%$. There was no maternal complications in booked placenta previa cases only there were very few complications in emergency cases. In abruptio placenta group also complications were more in emergency cases, irregular booked and uncontrolled pregnancy pathology cases.

\section{Recommendations}

Recommendation of our study is that maternal and neonatal mortality and morbidity can be reduced by 
timely and proper management of the patient in a good tertiary care centre having good neonatal set up and facility of all superspeciality department.

So, we recommend that,

All the antenatal patients should have $100 \%$ antenatal care, diagnosis of placenta previa by USG and proper management at well equipped tertiary care centre for the prevention of APH. Emergency admission should be avoided by planning timely termination of pregnancy in placenta previa and uncontrolled pregnancy pathology irrespective of gestational age to avoid maternal complications. Timely marriage and early planning of pregnancy to avoid infertility and ART procedures, which are newer emerging predisposing factors.

Funding: No funding sources

Conflict of interest: None declared

Ethical approval: The study was approved by the Institutional Ethics Committee

\section{REFERENCES}

1. Special Bulletin On Maternal Mortality In India 2016-18.

2. WHO, UNICEF, UNFPA and The World Bank, Trends in Maternal Mortality: 2000 to 2017 WHO, Geneva, 2019.

3. Textbook of Obstetrics - D.C. Dutta 9th edition "Safe motherhood, Epidemiology of Obstetrics; 562.

4. Practical Guide to High Risk: Pregnancy and Delivery, Fernando Arias. "Antepartum Haemorrhage"; 151.
5. Textbook of Obstetrics - D.C. Dutta 9th edition "Antepartum Haemorrhage "; pg. 228.

6. Textbook of Obstetrics - D.C. Dutta 9th edition "Antepartum Haemorrhage "; pg. 238.

7. Williams Obstetrics; 25th edition "Obstetrical Haemorrhage" pg. 769

8. Wasnik SK, Naiknaware SV. Antepartum haemorrhage: causes \& its effects on mother and child: an evaluation. Obstet Gynecol Int J. 2015;3(1):255-8.

9. Majumder S. Study of foetomaternal outcome of antepartum haemorrhage in pregnancy 1 . Int J Reprod Contracept Obstet Gynecol. 2015;4(6):1936-9.

10. Tyagi P, Yadav N, Sinha P, Gupta U. Study of antepartum haemorrhage and its maternal and perinatal outcome. Int J Reprod Contracept Obstet Gynecol. 2016;5(11):3972-7.

11. Kapadia LD, Dhrangiya B. Study of Maternal and Perinatal Outcome in 100 Cases of Abruptio Placentae International J Medic Res Health Sci. 2017;6(7):84-8.

12. Lakshmipriya. Study of maternal and fetal outcome In antepartum haemorrhage. master thesis. 2017:120

13. Kavita, Chavda R. Maternal and perinatal outcome in antepartum haemorrhage Hospital, southwestern Nigeria, J Med Sci. 2011;2(12):1243-7.

Cite this article as: Ghanchibhai F, Goyal S, Sharma N, Pargee A. Study of fetomaternal outcomes in antepartum haemorrhage at tertiary care centre. Int J Reprod Contracept Obstet Gynecol 2021;10:1509-14. 